\title{
A Study on Correlation of Traffic Accident Tendency with Driver Characters Using In-Depth Traffic Accident Data
}

\author{
Lin $\mathrm{Hu},{ }^{1,2}$ Xingqian Bao, ${ }^{1}$ Hequan $W u,{ }^{1,2}$ and Wenguang $W u(\mathbb{D})$ \\ ${ }^{1}$ School of Automotive and Mechanical Engineering, Changsha University of Science and Technology, Changsha 410114, China \\ ${ }^{2}$ Hunan Province Key Laboratory of Safety Design and Reliability Technology for Engineering Vehicle, \\ Changsha University of Science and Technology, Changsha 410114, China
}

Correspondence should be addressed to Wenguang Wu; wuwenguanglq@126.com

Received 24 December 2019; Accepted 27 March 2020; Published 2 June 2020

Academic Editor: Feng Chen

Copyright (C) 2020 Lin Hu et al. This is an open access article distributed under the Creative Commons Attribution License, which permits unrestricted use, distribution, and reproduction in any medium, provided the original work is properly cited.

\begin{abstract}
Traffic accidents are often related to the driver's driving behavior, which is mainly decided by his or her characters. In order to explore the correlation of traffic accident risk with driver characters, the age, driving experience, and driving style were statistically analyzed based on the China In-Depth Accident Study (CIDAS) database. Taking the number of casualties in the accident as evaluation indicators, the grey cluster analysis was used to classify the drivers into four accident risk ranks: low, medium to low, medium to high, and high. The results show that drivers aged 18-30 years are more likely to induce accidents; drivers with 6-10 years of driving experience have the highest risk to accidents, followed by drivers with 4-5 years of driving experience; and the driving style is also highly correlated with accident risk tendency.
\end{abstract}

\section{Introduction}

In China, especially in urban roads, due to mixed traffic flows, the traffic situation is more complicated. Although the accident rate is decreasing year by year, the number of casualties is still high every year [1]. For example, it is reported 0.9 per cent year-on-year decrease for 2018 in China, but there were still 166,906 traffic accidents in cars, which killed 46,161 people and injured 169,046 people. In addition, according to the reports of traffic accidents, nearly $90 \%$ of accidents are caused by human factors, and the human becomes one of the most unstable factors in causing traffic safety problems [2]. Traffic accidents are often related to the driver's driving behavior, which is mainly decided by his or her characters, such as the age, driving experience, and driving style. Drivers' willingness to reflect the driving style characteristics is based on their own driving ability, danger, and emergency handling attitude [3,4]. It is particularly urgent to study the correlation of traffic accident risk tendency with driver characters.

Accident Tendency Theory is one of the ancient and most widely known accident causation theories, which holds that accidents are indeed related to human personality [5]. In addition, from the statistical point of view, a certain type of driver would show more likely to be accident-prone than other drivers under the same driving environment conditions, which can be called the driver's accident tendency. In other words, accident propensity refers to individuals who have the characteristics of accident-prone among drivers [6].

The Grey System Theory was founded in 1982 by the Chinese scholar Professor Deng Julong. It is a systematic subject with the uncertainty system, which is mainly used to study the "small sample uncertainty problem" and describes the versatile factors that are difficult to describe quantitatively $[7,8]$. The generation and development of known information is used to extract valuable information and achieve a correct description of the system's operational behavior, evolutionary rules, and effective monitoring [9]. The problem of traffic accident risk with driver characters is not only complicated but also changes with time and environment. It can be summarized as follows: the system factors are not completely clear, the relationship between factors is not completely clear, the system structure is not fully known, and the principle of the system is not 
completely clear, that is to say, the system is an "information incomplete" system. Therefore, the system consisting of people and vehicles is a typical grey system, which is suitable for the grey system theory [10].

Exploring the correlation of traffic accident risk with driver characters, such as the age, driving experience, and driving style, would be an effective way to improve the traffic safety [11]. Based on the statistics of the actual traffic accident cases occurred from 2014 to 2016 in the CIDAS database, this paper analyzes the characters of drivers involved in these accidents, and the grey cluster analysis was used to classify the drivers into four accident risk ranks. This study would provide a theoretical basis for improving the domestic traffic design and standardizing the driving behavior of drivers.

\section{Data and Methods}

2.1. Data. The China In-Depth Accident Study (CIDAS) project was launched on July 15, 2011, by China Automotive Technology Research Center and several well-known automobile enterprises. It aims to provide basic data support and technical services for the automobile industry through in-depth investigation, analysis, and research on road traffic crashes in China [12].

356 crash cases are selected from the road vehicle accidents of the CIDAS project from 2014 to 2016 based on the following sampling criteria: (1) passenger car involvement, (2) detailed records of driver characters, (3) detailed injury records, (4) clear causation of the accident, and (5) age of the drivers ranging between 18 and 60 years.

2.2. Methods. A grey cluster evaluation model is established to classify the drivers' accident risk rank, and the main steps are as follows:

(1) Giving the cluster whitening number: select $n$ clustering objects, get $m$ clustering indicators, and construct an $n \times m$ matrix.

(2) Inputting the cluster whitening number into the computer, entering the grey clustering analysis and evaluation algorithm, including the clustering whitening number to be averaged and dimensionless; determining the grey class where each clustering index value belongs to each clustering object; using the valuation method or interpolation method to obtain the whitening weight function value of each grey class; calibrating the clustering weight grey number matrix; and constructing the clustering matrix.

(3) Performing grey evaluation based on the result of step (2).

2.2.1. Definition of Grey Cluster Objects and Indicators. The numbers of minor injuries, serious injuries, and deaths in the accident are selected as the evaluation indicators, and then the evaluation index matrix is constructed according to the following equation:

$$
X_{m \times n}=\left[\begin{array}{ccc}
9 & 4 & 5 \\
10 & 4 & 0 \\
4 & 2 & 0 \\
\vdots & \vdots & \vdots \\
1 & 0 & 0
\end{array}\right],
$$

where $m$ represents the number of the evaluation object, $m \in\{1,2,3, \cdots, 47\} ; n$ is the evaluation index, $n \in\{1,2,3\}$; and $n=1,2,3$ represents the number of minor injuries, serious injuries, and deaths in the accident, respectively. The range method is used to measure the indicators without dimension, shown as equation (2):

$$
X_{m n}=\frac{x_{m n}^{\prime}-x_{\min }}{x_{\max }-x_{\min }},
$$

where $x_{m n}^{\prime}$ is the elements in the matrix and $x_{\min }$ and $x_{\max }$ are the minimum and the maximum element in the matrix, respectively. The evaluation matrix after dimensionless processing is shown in the following equation:

$$
X_{m \times n}=\left[\begin{array}{ccc}
0.9 & 0.4 & 0.5 \\
1.0 & 0.4 & 0 \\
0.4 & 0.2 & 0 \\
\vdots & \vdots & \vdots \\
0.1 & 0 & 0
\end{array}\right] .
$$

2.2.2. Determining of Ash and Whitening Values. The driver accident risk rank is classified into four levels: low, medium to low, medium to high, and high. It is represented by the grey class $k=1,2,3$, and 4 , respectively, and then the evaluation matrix after dimensionless treatment is used to perform the cumulative frequency analysis [13, 14]. The whitening values of the four levels of the driver's risk tendency are represented by $\lambda_{n}^{1}, \lambda_{n}^{2}, \lambda_{n}^{3}, \lambda_{n}^{4}$, which are the relative values of the cumulative frequencies of the curves of $15 \%$, $40 \%, 60 \%$, and $85 \%$, respectively. The matrix of $\lambda$ is described in the following equation:

$$
\lambda=\left(\lambda_{n}^{1}, \lambda_{n}^{2}, \lambda_{n}^{3}, \lambda_{n}^{4}\right)=\left[\begin{array}{cccc}
0.1 & 0.1 & 0.3 & 0.4 \\
0 & 0 & 0.1 & 0.3 \\
0 & 0 & 0 & 0.2
\end{array}\right]
$$

\subsubsection{Construction of the Grey Class Whitening Weight} Function. The segmentation function can be used to construct the whitening weight function of the driver's risk tendency of inducing an accident [15-17]. The symbol $f_{n}^{k}$ indicates the whitening weight function of the $n$th clustering evaluation index, which belongs to subclass $K$. The whitening weight function of the indicator corresponding to minor injury, severely injury, and death is $f_{1}^{k}, f_{2}^{k}$, and $f_{3}^{k}$, respectively. Taking the minor injury indicator $f_{1}^{k}$ for 
example, the whitening weight function of four levels of risk tendency can be obtained from equations (5) to (8)

$$
\begin{aligned}
& f_{1}^{1}= \begin{cases}1, \quad x \in[0,0.1], \\
0, \quad x \in(0.1,1],\end{cases} \\
& f_{1}^{2}= \begin{cases}0, & x \notin[0.1,0.3], \\
\frac{0.3-x}{0.3-0.1}, & x \in[0.1,0.3],\end{cases} \\
& f_{1}^{3}= \begin{cases}0, & x \notin[0.1,0.4], \\
\frac{x-0.1}{0.3-0.1}, & x \in[0.1,0.3], \\
\frac{0.4-x}{0.4-0.3}, & x \in(0.3,0.4],\end{cases} \\
& f_{1}^{4}= \begin{cases}\frac{0.0 .3}{0.4-0.3}, & x \in(0.3,0.4], \\
\frac{x-4}{1,} & x \in(0.4,1] .\end{cases}
\end{aligned}
$$

Then, the clustering weight will be determined by the following equation:

$$
\eta_{n}^{k}= \begin{cases}\frac{\lambda_{n}^{k}}{\sum_{n=1}^{3} \lambda_{n}^{k}} \cdot\left(1-\frac{b}{i}\right), & \lambda_{j}^{k} \neq 0, \\ \frac{1}{i}, & \lambda_{j}^{k}=0,\end{cases}
$$

where $\eta_{n}^{k}$ and $\lambda_{n}^{k}$ are the clustering weight and whitening value, respectively, of the $n$th evaluation index in the $k$ th grey category and $b$ is the number of $\lambda_{n}^{k}$, which is equal to $0, i=3$.

According to equation (9), the grey cluster weight matrix of each evaluation index can be obtained for each subclass. Then, the reanalysis of grey clustering will be performed, the grey cluster evaluation value $\sigma_{m}^{k}$, which means the cluster evaluation value of the $m^{\text {th }}$ evaluation object in the $k^{\text {th }}$ grey class can be determined by the following equation:

$$
\sigma_{m}^{k}=\sum_{n=1}^{3} f_{m}^{k} X_{m}^{n} \eta_{n}^{k} .
$$

The cluster evaluation value sequence of the evaluation object $m \sigma_{m}^{k}=\left(\sigma_{m}^{1}, \sigma_{m}^{2}, \cdots, \sigma_{m}^{k}\right)$ will be used to identify the accident tendency level of $m$.

\section{Results}

3.1. Characters of Accident Vehicles Drivers. Traffic accidents are often related to the driver's driving behavior, which is mainly decided by his or her characters, and age, driving experience, and driving style are the main characters related with the handling performance [18-22].

3.1.1. Age. The drivers were divided into four age groups: 18 to 30 years old, 31 to 40 years old, 41 to 50 years old, and 51 to 60 years old. Figure 1 shows the age distribution of the drivers in traffic accident vehicles.

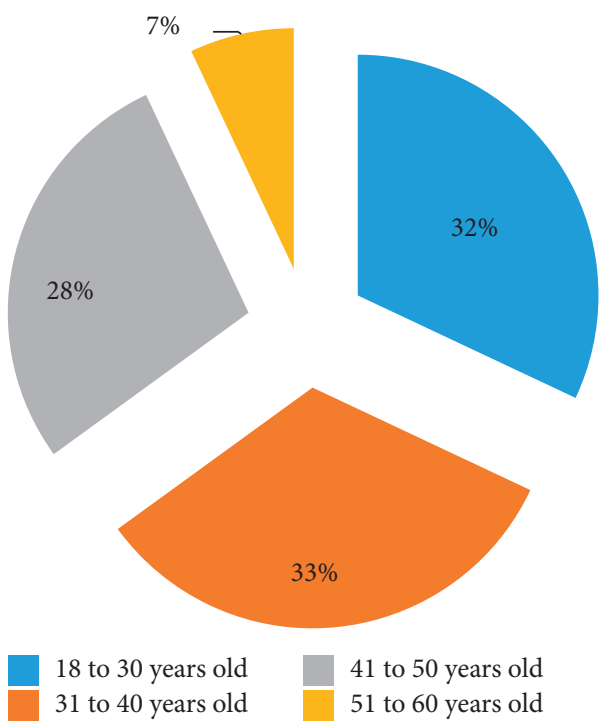

FIgURE 1: Age distribution of the drivers in traffic accident vehicles.

Figure 2 shows the number of casualties caused by different age groups.

Drivers aged 31-40 years account for the maximum number of traffic accidents, while drivers aged 51-60 years have the minimum number of traffic accidents and they also have the lowest casualties. Drivers in the $41-50$ age group have the highest number of fatal accidents, followed by drivers in the 51-60 age group. The visual characteristics, response time, speed estimation accuracy, and operation ability of drivers would change with the increase of age to a certain extent [23-25], so age would consequently influence a driver's accident risk tendency.

3.1.2. Driving Experience. Driving experience is an objective evaluation index related with the driver's driving ability [26-29]. In this study, the drivers were divided into six groups based on their driving experience: 3 years and below, 4 to 5 years, 6 to 10 years, 11 to 15 years, 16 to 20 years, and 20 years and above. Figure 3 shows the distribution of driving experience of drivers in accidents.

It can be seen that the drivers in the 6-10-year driving experience group contribute the most number of accidents, followed by the drivers in the 3-year driving experience group, and the drivers with 20 years or more driving experience account for the lowest number of accidents.

3.1.3. Road Types. Figure 4 shows the distribution of the road types where the accident occurred. In this study, four kinds of road types are involved: straight, curved road, intersection, and crossroad. The accidents occurred on the straight road account for the highest proportion (44.45\%) in the total accidents, followed by intersections and crossroads. If we consider the integration of data at intersections and crossroads, the number of accidents at intersections is more than half of the total number of accidents, which is consistent with other literatures [30-33]. 


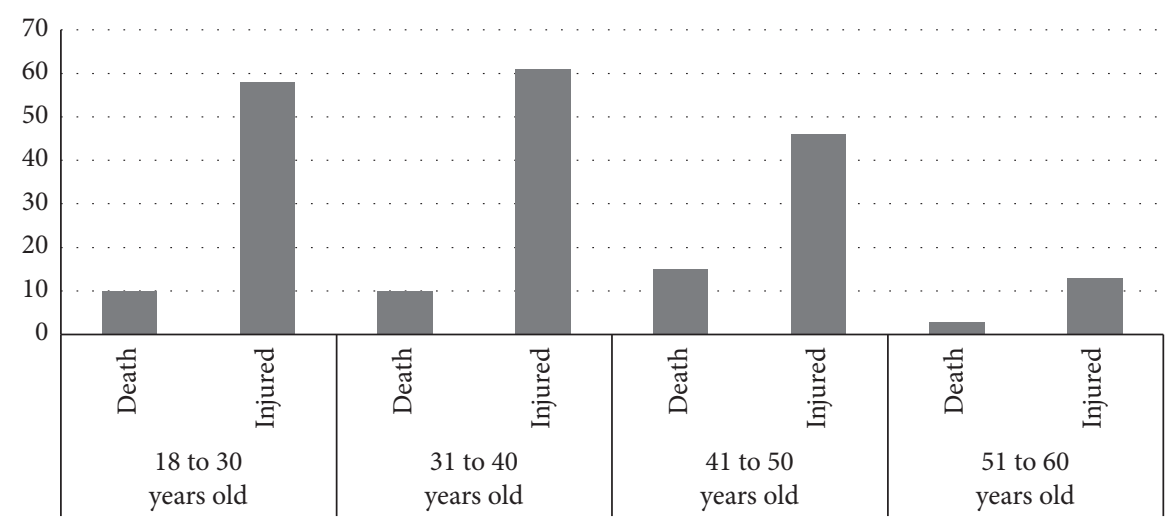

Figure 2: Casualties caused by drivers of different ages.

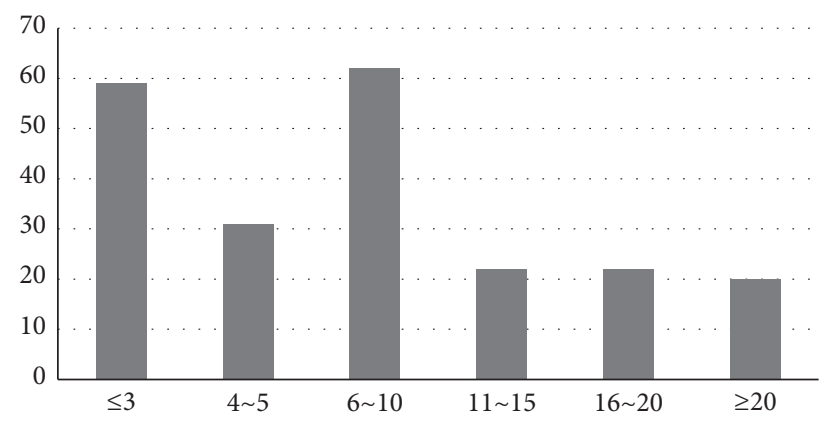

Figure 3: Distribution of driving experience of drivers in accidents.

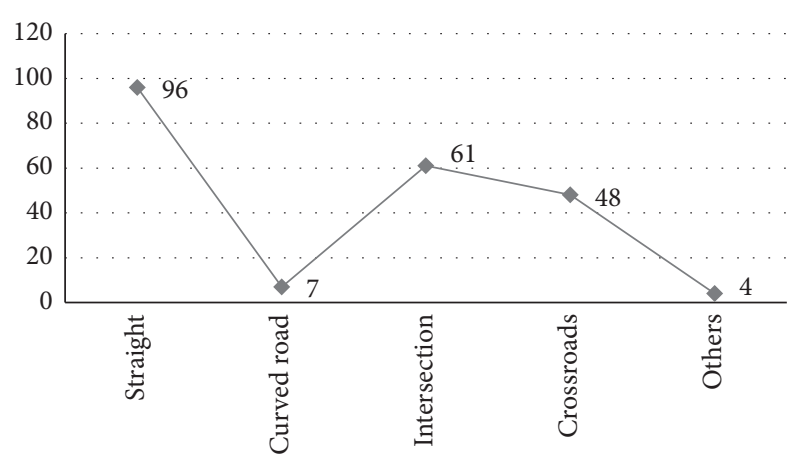

Figure 4: Distribution of road types in accidents.

3.1.4. Driving Style. Due to the significant deviation of the driver's gender in data samples, the accident cases involved female driver only accounts for $7.5 \%$ of the total sample, and the correlation of the driver's driving style with accident risk tendency was analyzed without gender-specific.

Figure 5 shows the distribution of driving styles of the accident vehicle drivers, which were recorded for each accident case in the CIDAS database, but the driving styles were declared by the driver himself. In the CIDAS database, the driving styles include three types that are adventure style, conservative style, and general style [34].

It can be seen from Figure 5, the general style and conservative style should be responsible for most accidents, which were not agreed with common recognition that the

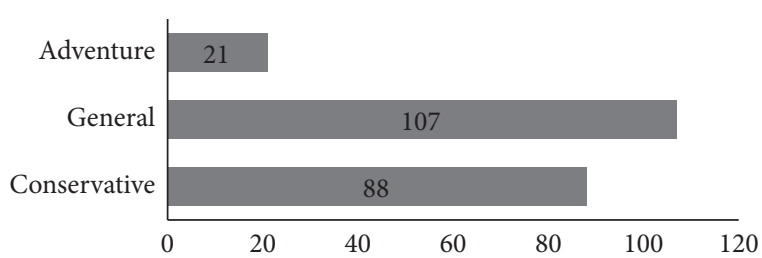

FIgURE 5: Distribution of driving styles of drivers in accidents.

adventure style drivers will have higher accident rates [35-38].

\subsection{Analysis of Driver's Accident Risk Tendency}

3.2.1. Grey Cluster Objects and Indicators. The drivers were divided into 47 classification groups as the object of grey clustering based on their age, driving experience, and driving style. The nonexistent combination and the combination with zero evaluation index were eliminated, as shown in Table 1.

3.2.2. Assessment Value of Drivers' Accident Risk Tendency in Various Character Groups. Table 2 lists the grey clustering assessment value of drivers' accident risk tendency in various character groups, and the groups with high accident risk tendency are given in italic. 
TABle 1: Grouping of driver characteristics.

\begin{tabular}{|c|c|c|c|c|c|c|}
\hline \multirow{2}{*}{ Number } & \multicolumn{3}{|c|}{ Driver characters } & \multicolumn{3}{|c|}{ Evaluation index } \\
\hline & Age/year & Driving experience/year & Driving style & Slightly injured & Seriously injured & Death toll \\
\hline 1 & 18 to 30 years old & $\leq 3$ & Conservative & 9 & 4 & 5 \\
\hline 2 & 31 to 40 years old & $\leq 3$ & Conservative & 10 & 4 & 0 \\
\hline 3 & 41 to 50 years old & $\leq 3$ & Conservative & 4 & 2 & 0 \\
\hline & $\ldots$ & $\cdots$ & $\cdots$ & $\cdots$ & $\cdots$ & $\cdots$ \\
\hline 45 & 51 to 60 years old & $11 \sim 15$ & Adventure & 0 & 0 & 1 \\
\hline 46 & 41 to 50 years old & $16 \sim 20$ & Adventure & 3 & 0 & 0 \\
\hline 47 & 51 to 60 years old & $16 \sim 20$ & Adventure & 1 & 0 & 0 \\
\hline
\end{tabular}

TABLE 2: Grey clustering calculation results of accident tendency of each group with driver characteristics.

\begin{tabular}{|c|c|c|c|c|}
\hline \multirow{2}{*}{ Number } & \multicolumn{3}{|c|}{ Driver characters } & \multirow{2}{*}{ Anecdotal tendency } \\
\hline & Age/year & Driving experience/year & Driving style & \\
\hline 1 & 18 to 30 years old & $\leq 3$ & Conservative & Medium to high \\
\hline 2 & 31 to 40 years old & $\leq 3$ & Conservative & Medium to high \\
\hline 3 & 41 to 50 years old & $\leq 3$ & Conservative & Medium to high \\
\hline 4 & 18 to 30 years old & $4 \sim 5$ & Conservative & High \\
\hline 5 & 31 to 40 years old & $4 \sim 5$ & Conservative & Medium to high \\
\hline 6 & 41 to 50 years old & $4 \sim 5$ & Conservative & High \\
\hline 7 & 18 to 30 years old & $6 \sim 10$ & Conservative & Medium to low \\
\hline 8 & 31 to 40 years old & $6 \sim 10$ & Conservative & Medium to low \\
\hline 9 & 41 to 50 years old & $6 \sim 10$ & Conservative & High \\
\hline 10 & 31 to 40 years old & $11 \sim 15$ & Conservative & Medium to high \\
\hline 11 & 41 to 50 years old & $11 \sim 15$ & Conservative & Medium to low \\
\hline 12 & 51 to 60 years old & $11 \sim 15$ & Conservative & Low \\
\hline 13 & 41 to 50 years old & $16 \sim 20$ & Conservative & Low \\
\hline 14 & 51 to 60 years old & $16 \sim 20$ & Conservative & Medium to low \\
\hline 15 & 41 to 50 years old & $\geq 20$ & Conservative & Medium to low \\
\hline 16 & 51 to 60 years old & $\geq 20$ & Conservative & Low \\
\hline 17 & 18 to 30 years old & $\leq 3$ & General & High \\
\hline 18 & 31 to 40 years old & $\leq 3$ & General & Medium to high \\
\hline 19 & 41 to 50 years old & $\leq 3$ & General & Medium to high \\
\hline 20 & 18 to 30 years old & $4 \sim 5$ & General & High \\
\hline 21 & 31 to 40 years old & $4 \sim 5$ & General & Medium to low \\
\hline 22 & 41 to 50 years old & $4 \sim 5$ & General & Medium to low \\
\hline 23 & 51 to 60 years old & $4 \sim 5$ & General & Medium to low \\
\hline 24 & 18 to 30 years old & $6 \sim 10$ & General & High \\
\hline 25 & 31 to 40 years old & $6 \sim 10$ & General & High \\
\hline 26 & 41 to 50 years old & $6 \sim 10$ & General & High \\
\hline 27 & 51 to 60 years old & $6 \sim 10$ & General & Low \\
\hline 28 & 18 to 30 years old & $11 \sim 15$ & General & Low \\
\hline 29 & 31 to 40 years old & $11 \sim 15$ & General & Medium to high \\
\hline 30 & 41 to 50 years old & $11 \sim 15$ & General & Medium to low \\
\hline 31 & 31 to 40 years old & $16 \sim 20$ & General & Medium to low \\
\hline 32 & 41 to 50 years old & $16 \sim 20$ & General & Medium to high \\
\hline 33 & 51 to 60 years old & $16 \sim 20$ & General & Medium to low \\
\hline 34 & 31 to 40 years old & $\geq 20$ & General & Low \\
\hline 35 & 41 to 50 years old & $\geq 20$ & General & Medium to low \\
\hline 36 & 51 to 60 years old & $\geq 20$ & General & Medium to low \\
\hline 37 & 18 to 30 years old & $\leq 3$ & Adventure & High \\
\hline 38 & 41 to 50 years old & $\leq 3$ & Adventure & Medium to high \\
\hline 39 & 18 to 30 years old & $4 \sim 5$ & Adventure & Medium to high \\
\hline 40 & 31 to 40 years old & $4 \sim 5$ & Adventure & Medium to low \\
\hline 41 & 41 to 50 years old & $4 \sim 5$ & Adventure & Medium to high \\
\hline 42 & 18 to 30 years old & $6 \sim 10$ & Adventure & Medium to high \\
\hline 43 & 31 to 40 years old & $6 \sim 10$ & Adventure & Medium to low \\
\hline 44 & 41 to 50 years old & $6 \sim 10$ & Adventure & Medium to high \\
\hline 45 & 51 to 60 years old & $11 \sim 15$ & Adventure & High \\
\hline 46 & 41 to 50 years old & $16 \sim 20$ & Adventure & Medium to low \\
\hline 47 & 51 to 60 years old & $16 \sim 20$ & Adventure & Medium to high \\
\hline
\end{tabular}




\section{Discussion and Conclusions}

4.1. Age Characteristics of Drivers with High Accident Tendency. The age distribution of drivers with high accident risk tendency is mainly concentrated in the age group of 18 to 30 years old. The main accident causation is the driver's illegal driving behaviors, such as robbing road priority and overtaking, and these accidents usually occurred in rush hours. From the perspective of psychological characteristics, young people have strong aggressive psychology, blind selfconfidence, and possible expectation imbalance.

\subsection{Driving Experience Characteristics of Drivers with High Accident Tendency. From the perspective of the driving experience, the drivers with 6-10 years of driving experience have the highest risk tendency, followed by the drivers with 4-5 years of driving experience. Drivers who have just started driving for 3 years or less are more cautious in their mental preparation, and drivers with more than 10 years of driving experience can deal with all kinds of unexpected situations with all kinds of skills. While a driver who is not senior but has some proficiency in road traffic rules and driving skills will be more risky when overtaking, violating, and so on and consequently has a higher risk tendency to causing accidents.}

4.3. Driving Style Characteristics of Drivers with High Accident Tendency. It can be seen from the results that three driving styles of drivers have similar accident risk tendency, and the general drivers should be responsible for the most accidents, which was not agreed with the common recognition that the adventure style drivers will have higher accident rates. The main reasons for this result are as follows: first, the driving style was declared by drivers themselves, there may be subjective interference in the classification of the driver's driving style, people often misjudge themselves, or they do not consider the change of their driving style during their trip, for example, the irregular driving of other vehicles make them feel anger or confusion; second, the accident rate of three styles of drivers would be a better evaluating indicator to indicate the correlation of the driver's driving style with accident risk tendency.

What we need to point out here is that, in this study, only the numbers of drivers in different age groups, driving style groups, and road types are used in statistical analysis, but in fact, drivers in different age groups would have different exposure of driving. Younger or middle-aged drivers may have more traveling miles than teen or older drivers. The number of causalities per mile may be a better indicator than considering only the number of causalities, and different road types also take up different proportions of all the trips. If the exposure of different types can be considered, the importance of intersections could be better explained. But due to the lack of relevant data in the current database, these parameters cannot be obtained right now. Considering the important statistical significance of these parameters, we will further update the procedure of in-depth accident investigation and record more valuable data.
The above research results show that drivers aged 18-30 years are more likely to induce accidents; drivers with 6-10 years of driving experience have the highest risk to accidents; and the driving style is also highly correlated with accident risk. The research on the characters of drivers with high accident risk tendency is conducive to targeted education and prevention of accidents in the future driving training and management.

\section{Data Availability}

The data used to support the findings of this study have been deposited in the figshare repository. The link is https:// figshare.com/s/bd4d605f61a1bf71fba7.

\section{Conflicts of Interest}

The authors declare that there are no conflicts of interest.

\section{Acknowledgments}

This work was financially supported by the Hunan Province Natural Science Outstanding Youth Fund (No. 2019JJ20017) and the National Natural Science Foundation of China (No. 51875049/51705035).

\section{References}

[1] Ministry of Public Security, Annual Report on Road Traffic Accidents of the People's Republic of China, Traffic Management Bureau of the Ministry of Public Security, Beijing, China, 2015.

[2] J. W. Niu, X. M. Zhang, Y. P. Sun et al., "Research on driving behavior of drivers taking over autopilot vehicles in dangerous situations," China Journal of Highway and Transport, vol. 31, no. 6, pp. 272-280, 2018.

[3] L. Sun, R. S. Chang, Y. Dong et al., "Impact of driving experience on driver's driving style and traffic violations," Chinese Journal of Health Psychology, vol. 22, no. 2, pp. 222-224, 2014.

[4] S. Li, "Effect of risk behavior attitude on drivers' driving style," Chinese Journal of Health Psychology, vol. 24, no. 10, pp. 1458-1460, 2016.

[5] H. Q. Jin, Y. Song, S. L. Zhang et al., "Construction of control model for traffic accident prevention system based on accident orientation theory," Ergonomics, vol. 17, no. 1, pp. 73-77, 2011.

[6] H. Q. Jin, "Driving Suitability," Hefei, Anhui People's Publishing House, Hefei, China, 1995.

[7] F. Y. Dong, M. D. Xiao, B. Liu et al., "Analysis of the construction method of whitening weight function in grey system teaching," Journal of North China University of Water Engineering, Natural Science Edition, vol. 31, no. 3, pp. 97-99, 2010.

[8] J. B. Hu and X. T. Cao, "Analysis of driver characteristics of road traffic accidents," China Journal of Highway and Transport, vol. 22, no. 6, pp. 106-110, 2009.

[9] Z. D. Zhao, "Application and analysis of grey system theory in automobile engineering," Journal of Chongqing University of Technology (Natural Science), vol. 28, no. 3, pp. 10-16, 2014.

[10] S. Y. Yan, Weapon Equipment Ergonomics," Harbin, pp. 344-359, Harbin Institute of Technology Press, Weihai, China, 2009. 
[11] M. Q. Guan and Q. N. Gong, “Analysis of drivers' incidence tendency of construction vehicles based on grey clustering method," Highway, vol. 22, no. 11, pp. 182-187, 2017.

[12] L. Hu, X. Hu, J. Wan, M. Lin, and J. Huang, "The injury epidemiology of adult riders in vehicle-two-wheeler crashes in China, Ningbo, 2011-2015," Journal of Safety Research, vol. 72, pp. 21-28, 2020.

[13] H. M. Zhou, Y. Sun, and X. J. Xu, "Research on freedom lane change behavior of urban road vehicles based on random utility theory," Journal of Transportation Research, vol. 3, no. 2, pp. 9-16, 2017.

[14] L. Hu, Y. Zhong, W. Hao et al., "Optimal route algorithm considering traffic light and energy consumption," IEEE Access, vol. 6, pp. 59695-59704, 2018.

[15] L. Hu, J. OU, J. Huang, Y. Chen, and D. Cao, "A review of research on traffic conflicts based on intelligent vehicles," IEEE Access, vol. 8, pp. 24471-24483, 2020.

[16] P. Kumar, M. S. Bains, N. Bharadwaj et al., "Impact assessment of driver speed limit compliance behavior on macroscopic traffic characteristics under heterogeneous traffic environment," Transportation Letters The International Journal of Transportation Research, vol. 4, no. 3, pp. 1-12, 2018.

[17] W. Qi, R. Z. Wang, and L. Wang, "Driving risk detection model of deceleration zone in expressway based on generalized regression neural network," Journal of Advanced Transportation, vol. 2018, no. 6, Article ID 8014385, 8 pages, 2018.

[18] L. Hu, S. Y. Fang, and Q. Chen, "Research on parameters influence of automobile-two-wheel vehicle collision accident reconstruction based on orthogonal experiment," Automotive Engineering, vol. 38, no. 5, pp. 567-573, 2016.

[19] L. Hu, X. Hu, Y. Che et al., "Reliable state of charge estimation of battery packs using fuzzy adaptive federated filtering," Applied Energy, vol. 262, 2020.

[20] P. Yong, C. J. Fan, L. Hu et al., "Tunnel driving occupational environment and hearing loss in train drivers in China," Occupational and Environmental Medicine, vol. 76, no. 1, pp. 97-104, 2019.

[21] H. J. Hou, L. S. Jin, Z. W. Guan et al., "The influence of driving style on driving behavior," China Journal of Highway and Transport, vol. 31, no. 4, pp. 22-31, 2018.

[22] Y. L. Zhai, Y. Q. Jin, and H. F. Chen, "Fatigue characteristics of drivers of different ages based on EEG signal analysis," China Journal of Highway and Transport, vol. 31, no. 4, pp. 63-81, 2018.

[23] Z. Zhang, L. Zhang, L. Hu, and C. Huang, "Active cell balancing of lithium-ion battery pack based on average state of charge," International Journal of Energy Research, vol. 44, no. 4, pp. 2535-2548, 2020.

[24] H. Q. Wu, S. J. Kuang, and H. B. Hou, "Research on application of electric vehicle collision based on reliability optimization design method," International Journal of Computational Methods, vol. 16, no. 7, pp. 1-15, 2019.

[25] L. ZhengY. S. Chen et al., "Research on static distance of queuing vehicles at signalized intersection based on computer vision," Journal of Highway and Transportation Research and Development, vol. 16, no. 2, pp. 95-100, 2018.

[26] X. H. Zhao, J. Rong, and Z. Q. Zhang, Driving Behavior Simulation Experiment Platform and its Application Research, People's Communications Press, Beijing, China, 2013.

[27] J. W. Han, Z. Q. Liu, B. Gong et al., "Mechanism analysis of urban road traffic accidents based on bayesian network," Science and Technology Innovation and Application, vol. 6, no. 8, pp. 23-24, 2017.
[28] X. Yue, Y. Ding, H. Hu et al., Analysis on the Association between Driver's Macroscopic Characteristics and Accident Type of Urban Traffic Accidents, China Control Conference, Shenyang, China, 2017.

[29] H. P. Wang and S. Eli, "Fuzzy clustering evaluation of altitude suitability for driving in continuous driving time," Science Technology and Engineering, vol. 2, no. 10, pp. 319-324, 2017.

[30] F. Chen, M. T. Song, and X. X. Ma, "Investigation on the injury severity of drivers in rear-end collisions between cars using a random parameters bivariate ordered probit model," International Journal of Environmental Research and Public Health, vol. 16, no. 14, pp. 26-32, 2019.

[31] F. Chen and S. Chen, "Injury severities of truck drivers in single- and multi-vehicle accidents on rural highways," Accident Analysis \& Prevention, vol. 43, no. 5, pp. 1677-1688, 2011.

[32] R. Du, G. Qiu, K. Gao, and L. Hu, “Abnormal road surface recognition based on smartphone acceleration sensor," Sensors, vol. 20, no. 2, p. 451, 2020.

[33] X. Yu, B. Li, T. Zhang, C. Tan, and H. Yan, "Variable weight coefficient optimization of gearshift actuator with directdriving automated transmission," IEEE Access, vol. 8, pp. 4860-4869, 2020.

[34] K. Gao, S. Huang, F. Han, S. Li, W. Wu, and R. Du, "An integrated algorithm for intersection queue length estimation based on IoT in a mixed traffic scenario," Applied Sciences, vol. 10, no. 6, pp. 2078-2088, 2020.

[35] J. L. Zhang, X. Y. Wang, M. S. Wang et al., "Transition probability and prediction of vehicle driving tendency in three-lane dynamic environment," Journal of Transportation Systems Engineering, vol. 17, no. 1, pp. 82-90, 2017.

[36] Y. Kong, R. S. Chang, T. J. Liu et al., "A comprehensive predictive model of driver's subjective fatigue state and steering wheel angle for accident tendency," Ergonomics, vol. 24, no. 2, pp. 40-46, 2018.

[37] D. Yang, Y. Z. Zhang, Y. Q. Zhang et al., "Comparative study on traffic injuries between Chinese expressways and ordinary highways from 2004 to 2015," Journal of Third Military Medical University, vol. 39, no. 6, pp. 589-596, 2017.

[38] F. Chen, H. R. Peng, X. X. Ma et al., "Examining the safety of trucks under crosswind at bridge-tunnel section: a driving simulator study," Tunnelling and Underground Space Technology, vol. 92, p. 103034, 2019. 